\title{
Impacto económico-financiero de la aplicación de las NIIFS en las PYMES de la ciudad de Ambato.
}

Economic-financial impact of the application of IFRS in the SMEs of the city of Ambato.

Christopher Neptali López Samaniego. ${ }^{1}$ \& Silvia Guadalupe Naranjo Lozada. ${ }^{2}$

\section{Abstract. $\quad$ DOI: $\underline{\text { https://doi.org/10.33262/visionariodigital.v4i4.1449 }}$}

In July 2009, the IASB, (International Accounting Standards Boards) creates IFRS (International Financial Reporting Standards) for SMEs (Small and Medium Enterprises) or small IFRS. This creation aims to systematize its application in this business sector. This document evaluates the cost-benefit ratio of the IFRS implementation process in a sector of SMEs in the center of the country. It is understood that the adoption of the new accounting standards means economic adjustments and improvements in the quality of accounting information as well as difficulties in the accounting process of some companies. This research shows the results of the exploratory and documentary type applied to 560 SMEs in the city of Ambato from the application of IFRS, summarized in 25 commercial activities carried out by this type of entity. The study is based on the financial information reported by the (SCVS) Superintendence of Companies, Securities and Insurance of the years 2017 and 2018. The analysis of the Financial Statements presents a strategic vision for the administrators of those who from these results can establish actions to achieve business growth and long-term sustainability. The quantitative information of the accounting effects that have generated the adoption of international standards to the accounts analyzed reflects an average decrease of $0.32 \%$ as observed in the different indicators. The results allow us to affirm that the IFRS for SMEs emphasizes the importance of the financial analysis of the Balances that SMEs have according to the economic reality of each sector.

Keywords: NIIF, PYMES, Financial Statements, Financial Analysis, Companies.

\section{Resumen.}

En julio del 2009 la IASB, (International Accounting Standards Boards) crea las NIIF (Normas Internacionales de Información Financiera) para PYMES (Pequeñas y Medianas Empresas) o NIIFS pequeñas. Esta creación tiene el objetivo de sistematizar

\footnotetext{
${ }^{1}$ Universidad Tecnológica Indoamerica, Ambato, Ecuador, chrisnep@hotmail.com

${ }^{2}$ Universidad Técnica de Ambato, Ambato, Ecuador, dranaranjo@yahoo.com
} 
su aplicación en este sector empresarial. El presente documento evalúa la relación costo beneficio del proceso de implementación de las NIIFS en un sector de las PYMES del centro del país. Entendiéndose que la adopción de las nuevas normas contables significa ajustes económicos y mejoras en la calidad de la información contable así como también las dificultades en el proceso contable de algunas empresas. La presente investigación muestra los resultados de tipo exploratorio y documental aplicada a 560 PYMES de la ciudad de Ambato a partir de la aplicación de las NIIF, resumido en 25 actividades comerciales que realizan este tipo de entidades. El estudio se basa en la información financiera reportada por la (SCVS) Superintendencia de Compañías, Valores y Seguros de los años 2017 y 2018. El análisis de los Estados Financieros plantea una visión estratégica para los administradores de las quienes a partir de estos resultados pueden establecer acciones para lograr el crecimiento empresarial y sostenibilidad a largo plazo. La información cuantitativa de los efectos contables que han generado la adopción de normas internacionales a las cuentas analizadas refleja un decrecimiento promedio del $0.32 \%$ como se observa en los distintos indicadores. Los resultados permiten afirmar que la NIIF para Pymes hace hincapié en la importancia del análisis financiero de los Balances que disponen las PYMES después de aplicación de la normativa.

Palabras claves: NIIF, PYMES, Estados Financieros, Empresas, Análisis Financiero

\section{Introducción.}

Vivimos en un mundo social globalizado derivado de las disciplinas sociales internacionales y de las políticas de internacionalización de las empresas (Chonchol, 1998). El desarrollo y crecimiento empresarial ha influenciado en todos los aspectos que conllevan al adelanto corporativo y la necesidad de estandarizar los procesos empresariales (Becerra, 2010). La armonización contable brinda información financiera comparable para la sociedad ayudando y facilitando un análisis e interpretación de manera comprensible. El crecimiento acelerado de las PYMES y la gran amplitud de actividades comerciales que estas generan hacen que los datos contables que arrojan sean oportuna, uniforme y de simplicidad absoluta. Disponer de un flujo de información financiera hacen que los accionistas, inversionistas, proveedores, sector financiero y gubernamental generen $\mathrm{e}$ incrementen transacciones comerciales internacionales indispensables y necesarias para la integración de los países. La normativa internacional y la información globalizada de la situación de las entidades, han influenciado en la competitividad y en las nuevas teorías del comercio mundial. Hoy es necesario la estandarización de los procesos contables y el control interno de gestión para homologar la información financiera de distintos países. Esto facilitara la introducción a mercados multinacionales, la inversión extranjera y los procesos de auditoría internacional.

Los Estados Financieros pretenden satisfacer en forma específica y clara todas las necesidades de información más comunes de las empresas, dándose prioridad a las necesidades de inversores y acreedores, actuales y potenciales, definidos habitualmente como usuarios tipo. 
Fowler Newton expresa que "Se espera que la información contable ayude a los miembros de este grupo a evaluar: a) la capacidad del ente emisor de los informes contables para: 1) pagar sus obligaciones en tiempo y forma; 2) generar y distribuir ganancias a sus propietarios (si se tratase de un ente con fines de lucro); b) el origen, la naturaleza, las características y el rendimiento de los recursos empleados; c) la calidad de la gestión de la administración, especialmente en cuanto al manejo de la rentabilidad, la solvencia y la capacidad de crecimiento del ente." (Enrique Fowler Newton, 2018)

Las 35 secciones de las NIIF para PYMES, que en el proyecto de investigación se utilizaran como indicadores y como modelo de análisis para direccionar a este tipo de entidades, muestran la variación económica que tuvieron al momento de la implementación de las NIIFS. Algunas normativas remiten expresamente a la información contenida en los balances, los mismos que entre otras cuestiones pueden brindar información de:

1) Liquidación y distribución de utilidades a partir de la información de los balances aprobados.

2) Conformación del grupo de cuentas del activo y pasivo y su estructura a partir de los resultados obtenidos según los Estados Financieros.

3) Estructura de las cuentas que conforman el patrimonio según como indica la normativa NIIF.

En nuestro País existe un amplio criterio de opciones para la medición del patrimonio y para la determinación de resultados y exposición de información contable. Cualquiera de ellas que se puede elegir dará lugar a determinadas cuestiones y mediciones diferentes. Es propósito de esta investigación analizar algunas situaciones para las cuales las normas susceptibles de ser aplicadas por una PYME admiten soluciones distintas y las implicancias que se generan a partir de ellas.

La organización IASB constantemente generan y cambian los procesos para modificar las NIIF para las PYMES, tratando de simplificar de manera importante algunos métodos. En Ecuador Las Normas Internacionales de Información Financiera (NIIF) son de aplicación obligatoria desde el año 2010 para todas las empresas que reportan a la Superintendencia de Compañías Valores y Seguros. En los últimas años (2010 en adelante), en las compañías se ha observado un incremento en la aplicación, contable financiera, actividad que ha creado egresos financieros y cargas administrativas, generando inconformidad en la aplicación. El presente estudio tiene la finalidad analizar el crecimiento y/o decrecimiento en las (PYMES) en la ciudad de Ambato. La investigación y el análisis principal de la aplicación de las NIIFS en las PYMES, del centro del país, no solo se enfoca en ver el cumplimiento, sino más bien que va más allá de ello, que es ver los impactos financieros que tuvieron desde el uso de estas normas.

\section{Antecedentes.}

La incomparabilidad de la información financiera dificulta las decisiones en la administración de los negocios dentro del ámbito contable financiero. Decidir sobre cómo 
actuar frente a una adquisición, fusiones, escisión, crédito, o inversión en el mercado global obstaculiza por no disponer de una información financiera. La internacionalización de las transacciones comerciales, el crecimiento de las empresas, las fusiones y adquisiciones, el interés de estas de generar créditos de inversiones más diversificados, obligan a los organismos pertinentes a redactar estándares contables uniformes. Los estándares permitirán la comparabilidad de la información financiera a nivel mundial

La mayoría de las instituciones financieras operan en el ámbito internacional, ya sea mediante créditos o inversiones. Las entidades antes citadas colaboran con las PYMES haciendo uso constante de su servicio para tramitar capital, por lo que estas instituciones financieras deben contar con informes contables para con los que se puede establecer los indicadores que los avalen y las tasas de interés.

Las personas que utilizan los Estados Financieros ven primordial que la información financiera debe ser elaborada bajo la normativa NIIFS, en donde las PYMES como practica normal debe utilizar NIIF ya que no ha sido asimilada con profundidad. Entre los usuarios internacionales que utilizan los sistemas contables Los sistemas contables buscan intercambiar información financiera entre los beneficiarios, en donde su objetivo es entregar información adecuada y oportuna para la toma de decisiones. Mas de un siglo las normas de contabilidad vienen generando información pertinente (AICPA, 2013), la misma le permite al usuario obtener datos eficientes, aplicando el análisis la identificación, y la medición, a las empresas que cumplen con la meta de otorgar datos efectivos y confiables. Con el transcurso de los años, cada uno de los países ha implementado organismos contables que ha permitido establecer un sistema de eficiente entre los organismos de control y los contribuyentes.

Los mercados de valores se internacionalizan, de la misma manera los productos y servicios han obtenido, la necesidad de utilizar un sistema contable uniforme que le permita de alguna manera dar a conocer información financiera global. Las NIIF para PYMES han sido creadas para unirse con el objetivo de unir esfuerzos que coincidan de manera contable a nivel universal. Las pequeñas y medianas empresas tienen la obligación de iniciar a utilizar las NIIF adoptándolas de manera planificada y clara

Es importante señalar que el hecho de cambiar de las NEC a NIIF; se deben redefinir las normas sobre contabilidad, los sistemas y procesos internos, la valuación de activos y pasivos y el recurso humano que pueden afectar también a las otras áreas de la organización, por lo tanto, las empresas tendrán costos y efectos de largo alcance, que deben ser medidos de acuerdo a la sección 35 de transición a la NIIF para las PYMES. En nuestro país Ecuador, el organismo de control es la Superintendencia de Compañías, quien expidió mediante Resolución No.SC.Q.ICI.CPAIFRS. del 27 de enero de 2011, la aplicación de la Norma Internacional de Información Financiera para Pequeñas y Medianas Entidades (NIIF para las PYMES), en donde se indica que debe realizar el registro, preparación y presentación de Estados Financieros, a partir del 1 de enero del 2012, todas aquellas compañías que cumplan las condicionantes señaladas en el artículo primero de la presente resolución. 


\section{Perspectiva Financiera.}

Las empresas deben contar con una estructura debidamente conformada en cada una de esas áreas, como es el caso de la financiera que desempeña un rol muy importante ya que son el motor que dinamiza la economía en todos los sectores productivos. (Ley de Compañías, 2017) Las empresas como las PYMES aportan un papel fundamental en el crecimiento de un país, por su capacidad para generar empleo, producción y recursos, por lo que se analizan algunos indicadores financieros que reflejan la situación económica de este grupo durante los años 2017 y 2018 agrupados en 25 sectores que presentaron los Estados Financieros ante la Superintendencia de Compañías, Valores y Seguros (SCVS). Las (SCVS) revisa las características e instrumentos de fomento productivo que se establecen en el Código Orgánico de la Producción, Comercio e Inversiones, en el cual se presentan los parámetros utilizados para dar a conocer el tamaño de las compañías de acuerdo a las directrices (Ley de Compañías, 2017) que a continuación se detalla.

Tabla N. 1 Clasificación de las PYMES.

\begin{tabular}{|c|c|c|c|c|}
\hline Variables & Micro Empresa & Pequeña Empresa & Mediana Empresa & Grandes Empresas \\
\hline Personal ocupado & De $1-9$ & De $10-49$ & De $50-199$ & $\geq 200$ \\
\hline Valor bruto de ventas anuales & $\leq 100.000$ & $100.001-1.000 .00$ & $01.000 .001-5.000 .000$ & $>5.000 .000$ \\
\hline Monto de activos & Hasta US\$ 100.000 & $\begin{array}{c}0 \text { De US\$ } 100.001 \\
\text { hasta US\$ } 750.000\end{array}$ & $\begin{array}{c}\text { De US\$ 750.001 } \\
\text { hasta US\$ 3.999.999 }\end{array}$ & $\geq 4.000 .000$ \\
\hline
\end{tabular}

Fuente: (Superintendencia compañías, valores y seguros , 2019)

\section{Metodología}

La investigación es de carácter indagatorio documental y aplicado a las PYMES de la ciudad de Ambato, los mismos que se encuentran clasificados en sectores específicos (Tabla $\mathrm{N}^{\circ} 2$ ) utilizando para ellos información secundaria Estados Financieros obtenido de la Superintendencia de compañías Valores y Seguros de los años 2017 y 2018. Sobre realidades empresariales es el enfoque de la presente investigación, ya que se aplicada a estudios con el fin de evaluar el impacto financiero por la aplicación de las NIIFS, con los cuales se han obtenido los resultados que se dan a conocer en el presente documento en donde se realiza un comparativo de los saldos de los Estados Financieros presentados al 31-12-2018. Además, se enfoca en un análisis de las variaciones económicas las que se evidencian en los balances presentados por de las Pymes, de la ciudad de Ambato. Además ayuda a diseñar y estructurar estrategias gerenciales que permitan fortalecer a las empresas a un lenguaje financiero común que le permitan encaminar por los beneficios económicos y la rentabilidad. En 560 PYMES registradas en la Superintendencia de Compañas Valores y seguros se aplicaron el análisis financiero, las mismas que se agrupa en 25 categorías. 
Tabla N. 2 Clasificación por sectores de las pequeñas y medianas empresas de Ambato

\begin{tabular}{ll}
\hline 1 & Industrias Manufactureras. \\
\hline 2 & Productos Alimenticios. \\
\hline 3 & Bebidas. \\
\hline 4 & Productos De Tabaco. \\
\hline 6 & Productos Textiles. \\
\hline 7 & Crendas De Vestir. \\
\hline 8 & Productos De Madera Y Corcho, Excepto Muebles \\
\hline 9 & Papel Y De Productos De Papel. \\
\hline 10 & Impresión Y Reproducción De Grabaciones.
\end{tabular}

11 Coque Y De Productos De La Refinación Del Petróleo.

\begin{tabular}{ll}
\hline 12 & Substancias Y Productos Químicos. \\
\hline 13 & Productos Farmacéuticos \\
\hline 14 & Productos De Caucho Y Plástico. \\
\hline 15 & Productos Minerales No Metálicos. \\
\hline 16 & Metales Comunes. \\
\hline 18 & Productos De Informática, Electrónica Y Óptica. \\
\hline 19 & Equipo Eléctrico. \\
\hline 20 & Maquinaria Y Equipo N.C.P. \\
\hline 21 & Vehículos Automotores, Remolques Y Semirremolques. \\
\hline 22 & Otros Tipos De Equipos De Transporte. \\
\hline 23 & Fabricación De Muebles. \\
\hline 24 & Otras Industrias Manufactureras. \\
\hline 25 & Reparación E Instalación De Maquinaria Y Equipo.
\end{tabular}

Fuente: (Superintendencia compañías, valores y seguros ,2019)

Autoría: Investigación propia.

\section{Fundamentos Teóricos}

\section{Definición de las PYMES}

En nuestro país se lama PYMES al conjunto de pequeñas y medianas empresas que, de acuerdo al número de trabajadores, volumen de ventas, años en el mercado, y sus niveles de producción, activos, pasivos tienen características similares en sus procesos de crecimientos. (Carrillo, 2019). Las PYMES difieren en tamaño, cantidad de ingresos, activos y número de empleados, principalmente. Estos pueden ser los indicadores para la definición de las PYMES, sin embargo, cada país define de acuerdo a sus criterios. La IASB define las PYMES como entidades que no tienen responsabilidad pública y divulgan Estados Financieros de uso general. (International Accounting Standards Board, 2012) El objetivo de los Estados Financieros es brindar información sobre la posición financiera, el rendimiento y el crecimiento que sea útil para la toma de decisiones a los usuarios que no exigen informes a la medida de sus necesidades específicas de información (International Financial Reporting Standar, 2018). 
La NIIF para las PYMES se ha desarrollado mediante:

a. La extracción de los conceptos fundamentales del Marco Conceptual del IASB y de los principios y guías obligatorios relacionados de las NIIF completas (incluyendo las interpretaciones), y

b. La consideración de las modificaciones apropiadas en función de las necesidades de los usuarios y las consideraciones de costo-beneficio de producirlas. (Normas Internacionales de Información Financiera, 2019)

Debido a la variedad de las PYMES, se habla de una estructura muy amplia y en diferentes contextos generándose un sin número de definiciones y/o criterio de status (micro, pequeña o mediana) según la cantidad de empleados, valor de activos e incluso el capital o cantidad de ventas con que dispongan. En todos los casos y pese a las dificultades y retos que enfrentan las Pymes, son generadores de empleo y del desarrollo económico en todos los países del mundo. Las PYMES juegan un papel sumamente fundamental para el incremento de los ingresos y la productividad incorporando tecnología, innovaciones y conocimiento a sus productos, así como mejoras en la gestión empresarial. (Stumpo, 2018)

\section{Las Pymes en el Mundo.}

Las Pymes representan el 50\% del PIB a nivel global, según la ONU (31 de julio de 2018) en España, las Pymes son el motor del crecimiento de la economía. El 99,84\% de las empresas españolas tienen menos de 250 trabajadores, de las cuales el 94\% son autónomos o microempresas de menos de 10 empleados, según datos ofrecidos por el Consejo Internacional para la pequeña empresa. (Dir\&ge, 2018)

El panorama internacional no es muy diferente y por eso la ONU ha destacado el papel que las microempresas juegan en la economía mundial. El organismo internacional ha puesto en valor su importancia en la reducción de la pobreza y fomento del desarrollo, especialmente en los países en crecimiento. (Acnur.org, 2019)

La ONU concibe esto como una oportunidad "Todas las empresas familiares son PYMES". ¡Cuántas veces habré oído esta afirmación! Seguramente, la confusión tiene que ver con el hecho de que la mayoría de empresas españolas son PYMES, sean o no de propiedad familiar. Fijaos que solo un 0,35\% de estas son grandes con más de 250 trabajadores, según revela el working paper. Demografía de la empresa española. El resto son microempresas (un 91\%), empresas pequeñas $(7,5 \%)$ y empresas medianas $(1,2 \%)$. Y esto no solo pasa en España, también sucede en Europa y Estados Unidos, donde la mayoría de empresas son microempresas o PYMES, y las grandes empresas son una minoría: un 0,5\% del tejido empresarial europeo y un $0,65 \%$ del estadounidense. Así que afirmar que "las empresas familiares son pequeñas" es tan impreciso como decir que "las empresas de Estados Unidos son grandes". (Garcia \& Callejas, 2019)

La radiografía del tejido empresarial europeo y norteamericano es muy similar. También en Europa y Estados Unidos predominan las PYMES y las grandes empresas son minoría, la 
investigación nos permite concluir que la mayoría de empresas familiares son PYMES porque la mayoría de empresas españolas son PYMES (Tápies, 2010)

\section{Las Pymes en Latinoamérica}

Según el estudio "Políticas para la inserción de las microempresas y las pequeñas y medianas empresas en cadenas globales de valor en América Latina" dice existen más microempresas es en el vecino país Perú, seguido por Ecuador y México. En el caso de las pequeñas empresas, Argentina ocupa el liderato y de las medianas empresas se encuentra en Uruguay.

Las MIPYMES de Latinoamérica son un pilar fundamental en la economía de los países, teniendo en cuenta su proporción con relación al número total de empresas, su contribución al empleo y en menor medida, su contribución al producto. Pero esta importancia disminuye cuando se las considera en cuanto a la proporción de las exportaciones nacionales que corresponden a las MIPYMES y la productividad de éstas (Stezano, 2013). En la Tabla N 3 se presenta el detalle de las empresas por países consideradas en el estudio indicado, el cual, según el autor, define el tamaño de las microempresas y las pequeñas y medianas según las categorías empleadas por el Instituto Nacional de Estadística de cada uno de los países.

Tabla N. 3 Estadísticas de las PYMES

\begin{tabular}{lllll}
\hline País & Microempresas & $\begin{array}{l}\text { Pequeñas } \\
\text { Empresas }\end{array}$ & $\begin{array}{l}\text { Medianas } \\
\text { Empresas }\end{array}$ & $\begin{array}{l}\text { Grandes } \\
\text { Empresas }\end{array}$ \\
\hline Argentina & $81.60 \%$ & $16.10 \%$ & $1.90 \%$ & $0.40 \%$ \\
\hline Brasil & $85.40 \%$ & $12.10 \%$ & $1.40 \%$ & $1.10 \%$ \\
\hline Chile & $90.40 \%$ & $7.80 \%$ & $1.20 \%$ & $0.60 \%$ \\
\hline Colombia & $93.20 \%$ & $5.50 \%$ & $1.00 \%$ & $0.30 \%$ \\
\hline Ecuador & $95.40 \%$ & $3.80 \%$ & $0.60 \%$ & $0.20 \%$ \\
\hline México 9 & $95.40 \%$ & $3.60 \%$ & $0.80 \%$ & $0.20 \%$ \\
\hline Perú & $98.10 \%$ & $4 \%$ & $0.34 \%$ & $0.02 \%$ \\
\hline Uruguay & $83.10 \%$ & $13.20 \%$ & $3.10 \%$ & $0.60 \%$ \\
\hline
\end{tabular}

Fuente: (Stezano, 2013) “Políticas para la inserción de las microempresas.

\section{Las Pymes en el Ecuador}

Según la investigación realizada por diario el telégrafo, El 95\% de las empresas en el país son Pymes Redacción Economía - 28 de junio de 2017.

La mayoría de las PYMES se dedican a actividades de comercio. Cifras brindadas por la ministra de Industrias, Eva García, dan cuenta del papel protagónico que tienen las micro, pequeñas y medianas empresas dentro de la economía ecuatoriana. El 95\% del tejido empresarial son PYMES; de ellas, el $90 \%$ son micro, $8,6 \%$ son pequeñas, y $1.4 \%$ son medianas. La mayor parte actúa en el sector comercial con el $54 \%$, en manufactura están $36,4 \%$ y en servicios $9,5 \%$. A través de las PYMES se hacen el $64 \%$ de las compras públicas. Y, además, abarcan el 35\% de todas las ventas nacionales. (Diario el Telégrafo, 2017) 
Tabla N. 4 Conformación de las empresas en el Ecuador

\begin{tabular}{lcc} 
PAIS & VALOR & \% \\
\hline Microempresas & 26.475 .00 & $51.29 \%$ \\
\hline Pequeñas Empresas & 15.892 .00 & $30.79 \%$ \\
\hline Medianas Empresas & 6.494 .00 & $12.58 \%$ \\
\hline Grandes Empresas & 2.757 .00 & $5.34 \%$ \\
\hline \multicolumn{1}{c}{ Total } & $\mathbf{5 1 . 6 1 8}$ & $\mathbf{1 0 0 \%}$
\end{tabular}

Fuente: (Superintendencia compañías, valores y seguros , 2019)

\section{Selección de la Muestra}

La muestra seleccionada consiste en revisar los Estados Financieros y la información contable que arrojan los mismos tomando como base 9 indicadores esenciales de la muestra de 25 sectores clasificado por la Superintendencia de Compañía, Valores y Seguros de un total de 560 empresas PYMES de la ciudad de Ambato durante el periodo 2017 y 2018 . El estudio presenta un análisis comparativo entre estos dos periodos económicos. (Superintendencia compañías, valores y seguros ,2019)

Información que se revela a través de tablas y gráficos estadísticos generados en la investigación.

Tabla N. 5 indicadores Financieros

\begin{tabular}{l}
\hline Indicadores \\
\hline Liquidez Corriente \\
Prueba Acida \\
Endeudamiento Del Activo \\
Endeudamiento Patrimonial \\
Endeudamiento Del Activo Fijo \\
Apalancamiento \\
Apalancamiento Financiero \\
Rotación De Cartera \\
Rotación De Activo Fijo \\
Rotación De Ventas \\
Impacto Gto Adm. Y Ventas \\
Impacto Carga Financiera \\
Rentabilidad Neta Del Activo \\
Margen Bruto \\
Margen Operacional \\
Margen Neto \\
Rentabilidad Operacional Del Patrimonio \\
Rentabilidad Financiera
\end{tabular}

Fuente: Investigación propi 


\section{Resultados.}

Una vez se ha realizado los comparativos de los Estados Financieros antes y después de aplicar las NIIFS, lo resultados son claros y evidentes y reflejan un decrecimiento general, En el estudio se observa la variación de la cifras en las cuentas contables del grupo en mención, reflejando resultados no muy alentadores.

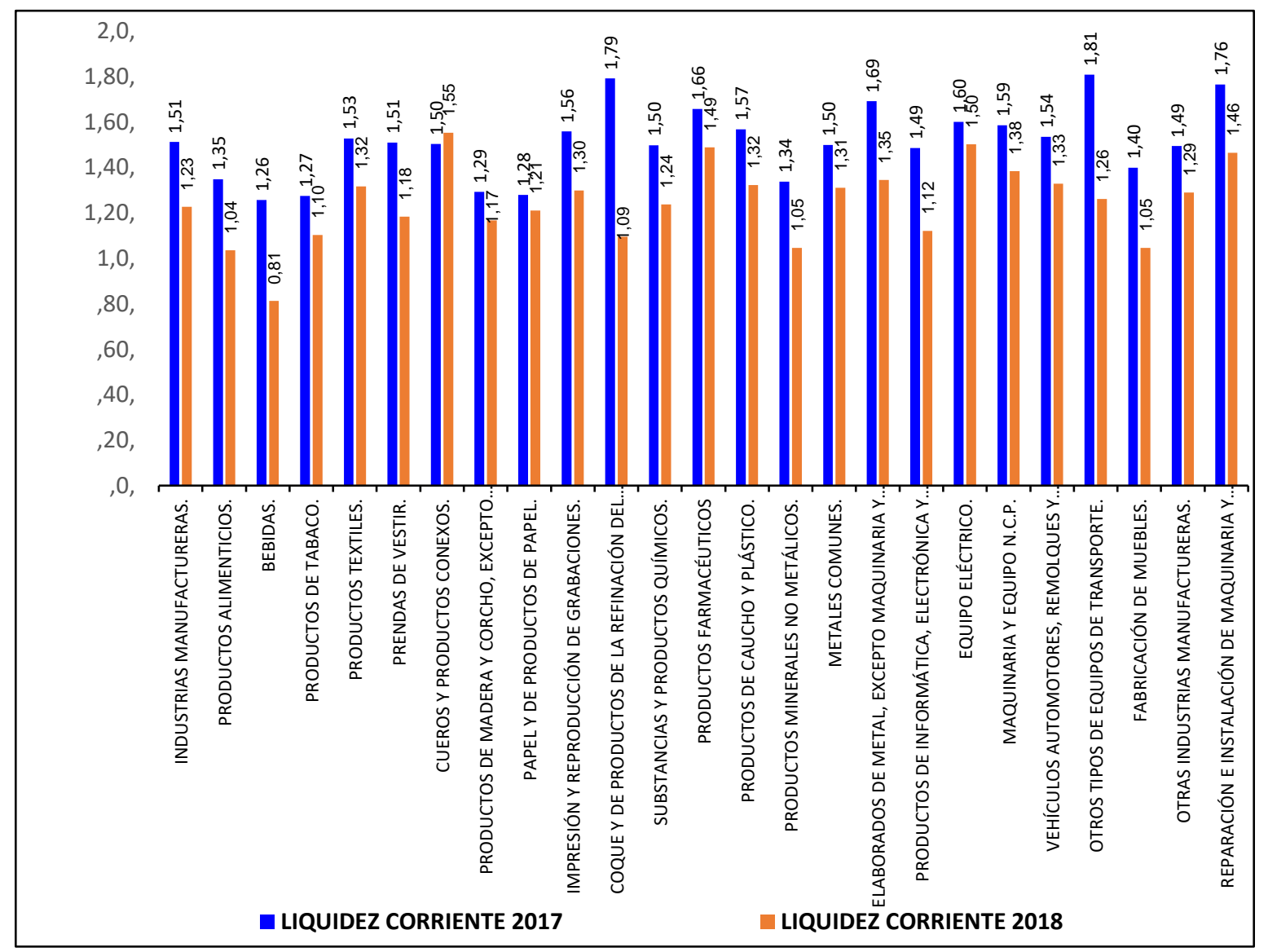

Gráfico $\mathbf{N}^{\circ} 1$ Liquidez Corriente

Fuente: Investigación propia

La liquidez corriente, surge de la necesidad de medir la capacidad que tienen las empresas para cancelar sus obligaciones de corto plazo. Sirven para establecer la facilidad o dificultad| que presenta una compañía para pagar sus pasivos corrientes al convertir a efectivo sus activos corrientes. Se trata de determinar qué pasaría si a la empresa se le exigiera el pago inmediato de todas sus obligaciones dentro de un año. Aplicado este indicador en los dos periodos años 2017 y 2018 refleja una perdida promedio de liquidez de $\mathbf{- 0 . 2 7 \%}$, obligando a que se realiza una restructuración financiera. (Superintendencia compañías, valores y seguros , 2019) 


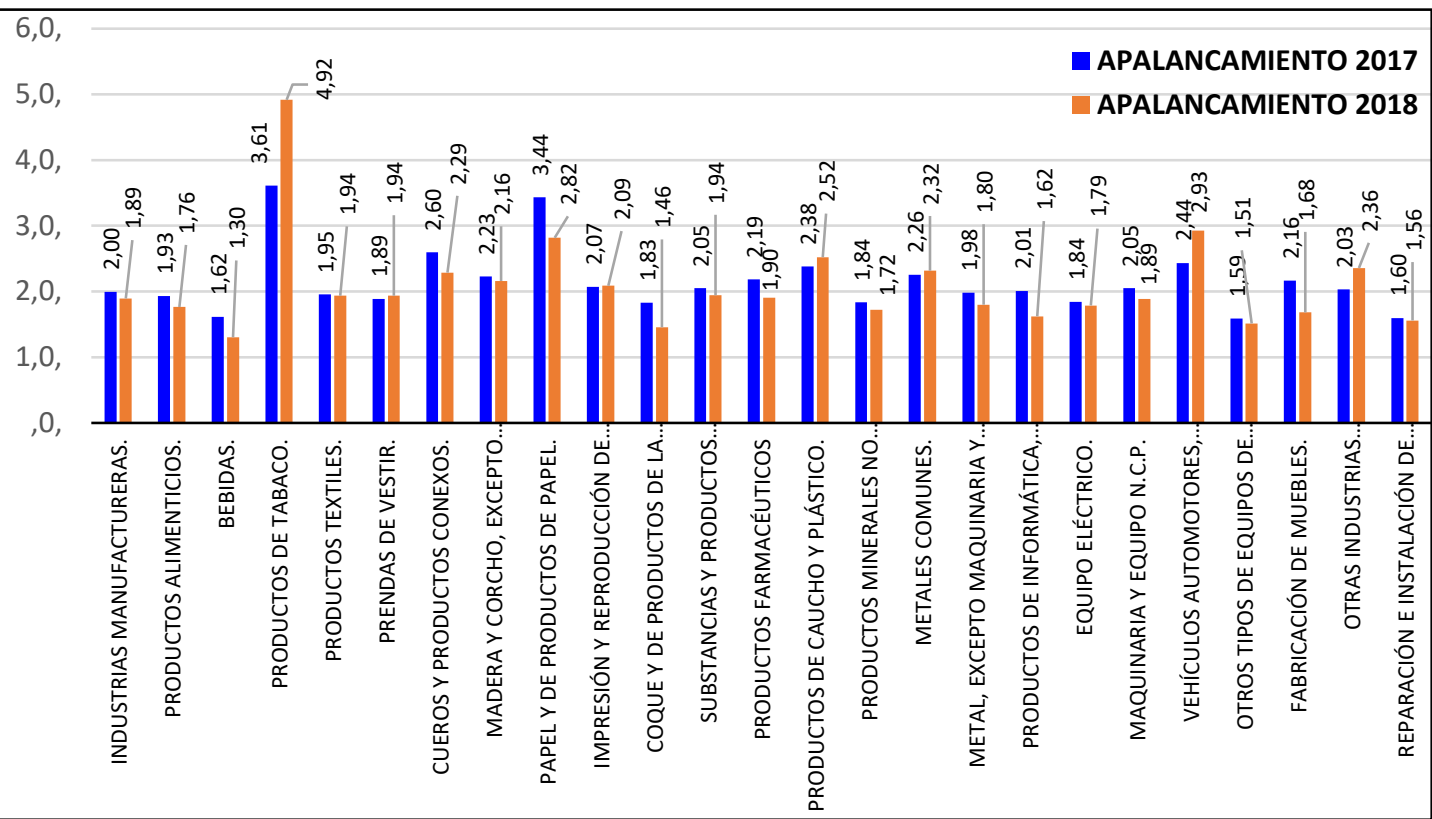

Gráfico $\mathbf{N}^{\circ} 3$ Apalancamiento

Fuente: Investigación propia.

El $0.06 \%$ promedio, Se interpreta como el número de unidades monetarias de activos que se han conseguido por cada unidad monetaria de patrimonio. Es decir, determina el grado de apoyo de los recursos internos de la empresa sobre recursos de terceros.

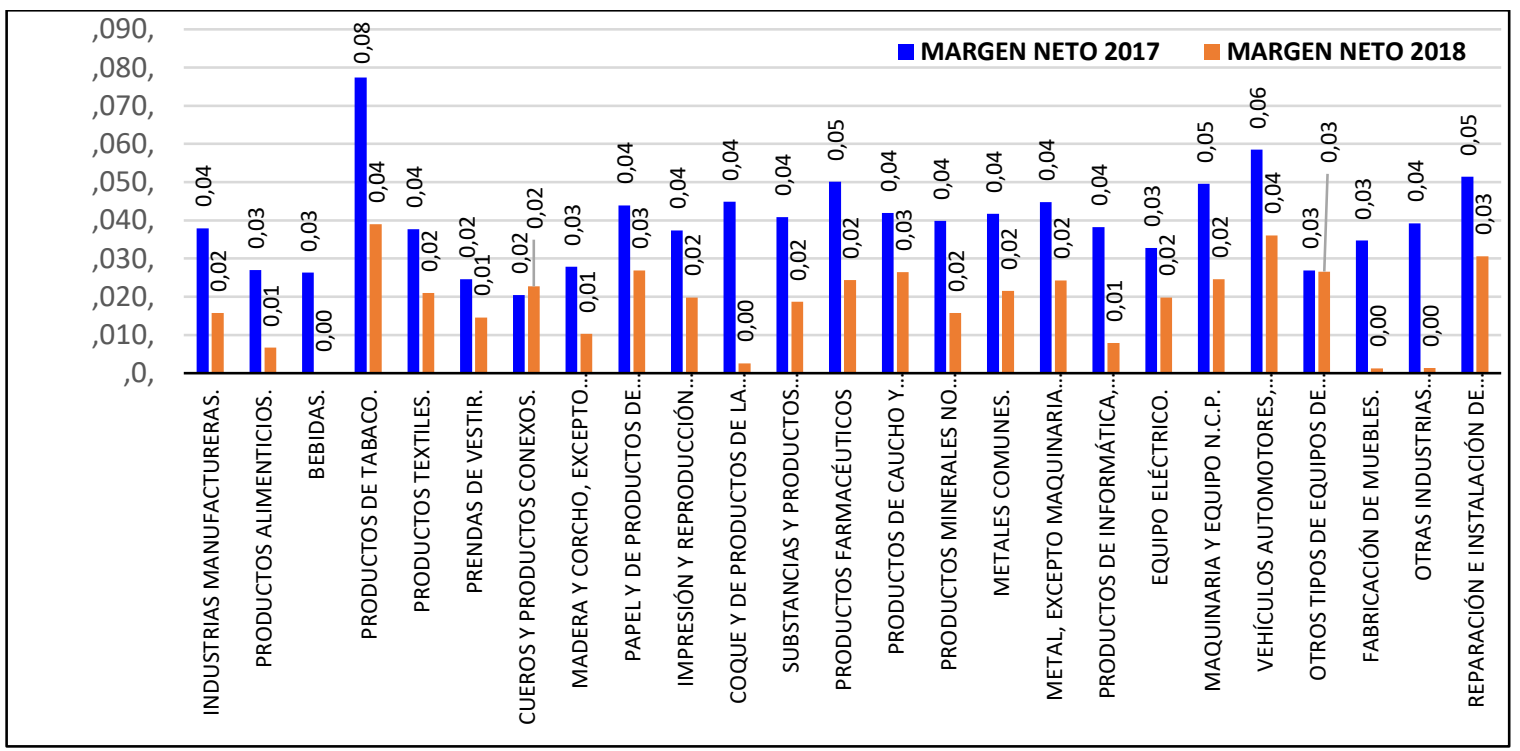

Gráfico $\mathbf{N}^{\circ} 6$ Margen Neto

Fuente: Investigación propia

Los índices de rentabilidad de ventas muestran la utilidad de la empresa por cada unidad de venta. En el presente análisis se obtuvo un $\mathbf{- 0 . 0 2}$ de rentabilidad, Se debe tener especial cuidado al estudiar este indicador, comparándolo con el margen operacional, para establecer si la utilidad procede principalmente de la operación propia de la empresa, o de otros ingresos diferentes. 


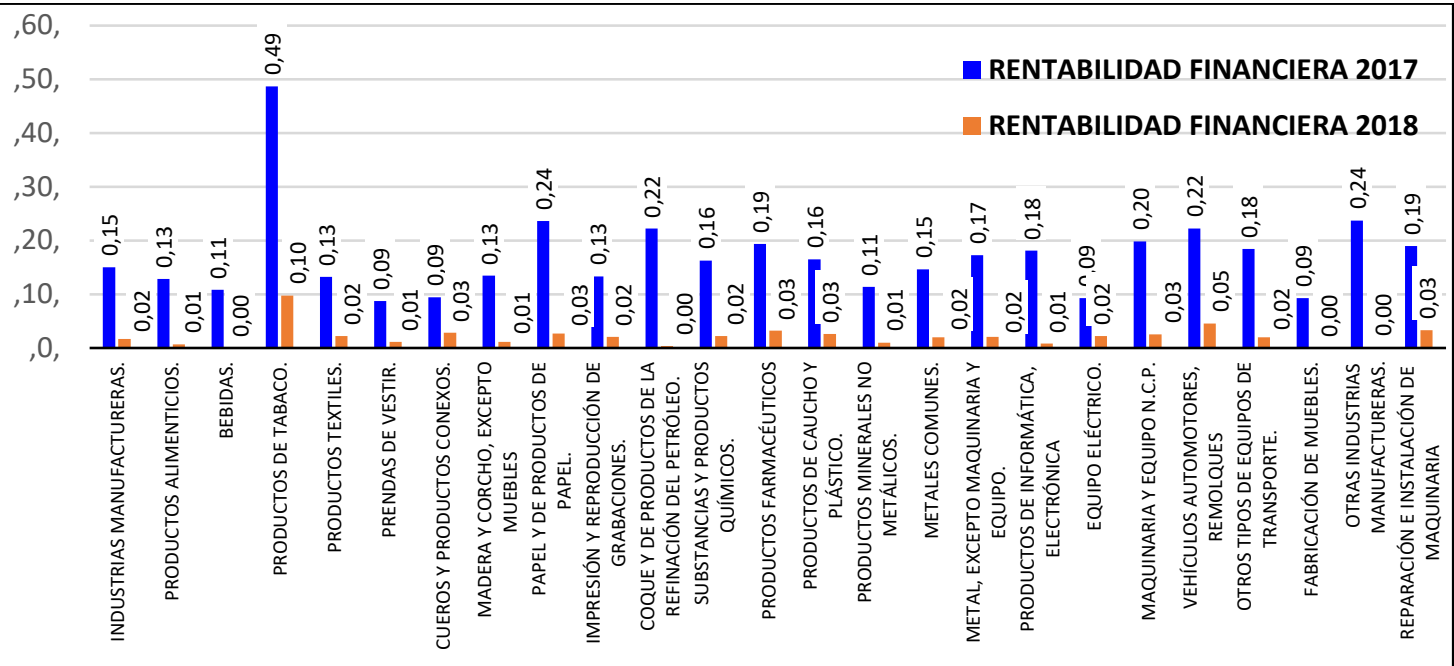

Gráfico $\mathbf{N}^{\circ} \mathbf{7}$ Rentabilidad Financiera

Fuente: Investigación propia.

Cuando un accionista o socio decide mantener la inversión en la empresa, es porque la misma le responde con un rendimiento mayor a las tasas de mercado o indirectamente recibe otro tipo de beneficios que compensan su frágil o menor rentabilidad patrimonial. De esta forma, la rentabilidad financiera se constituye en un indicador sumamente importante, pues mide el beneficio neto (deducidos los gastos financieros, impuestos y participación de trabajadores) generado en relación a la inversión de los propietarios de la empresa. En el comparativo se determina una disminución del $-0.15 \%$.

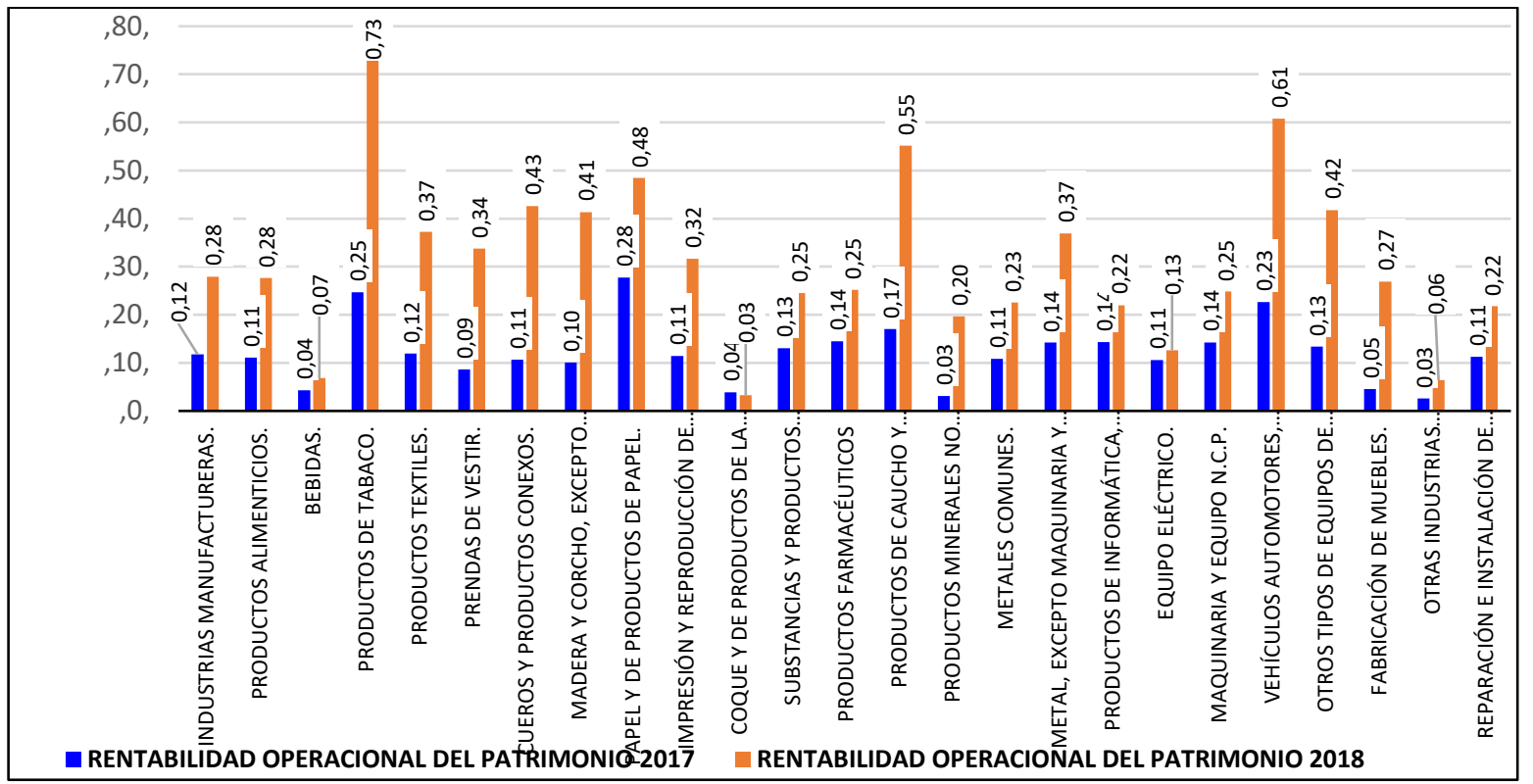

Gráfico $\mathbf{N}^{\circ} \mathbf{8}$ Rentabilidad Del Patrimonio

Fuente: Investigación propia

La rentabilidad operacional del patrimonio tuvo un incremento del $0.19 \%$, indicándonos la rentabilidad que le ofrece a los socios o accionistas el capital que han invertido en la empresa, sin tomar en cuenta los gastos financieros ni de impuestos y participación de trabajadores. 


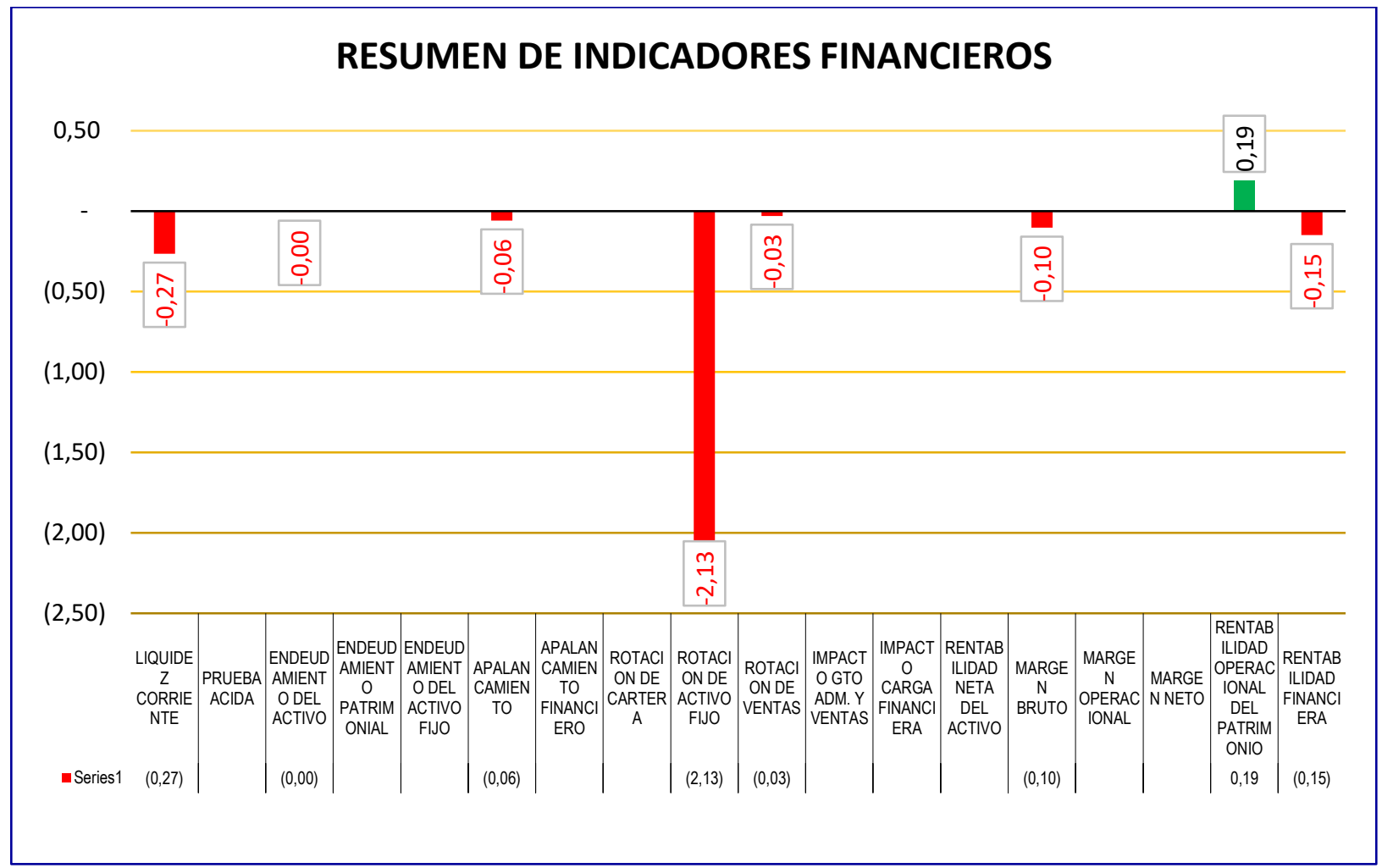

Gráfico $\mathbf{N}^{\circ} 9$ Resumen de Indicadores Financieros

Fuente: Investigación propia

Como un resumen general se puede interpretar que, el estudio determinó un decrecimiento del sector Pymes en un -0.32 comparados entre los periodos económicos 2017 y 2018. Destacando que el único indicador que arrojo cifras positivas fue el de Rentabilidad del Patrimonio. A partir de esta información los Gerentes de las empresas de este sector deberán tomar muy en cuentan estas cifras con el fin de contrarrestar y aplicar herramientas financieras que mejoren el crecimiento empresarial.

\section{Contribuciones.}

El análisis del comportamiento de las PYMES de la ciudad de Ambato permite recopilar datos de los Estados Financieros de los años 2017 y 2018. Además, se analiza las estadísticas de ciertos sectores específicos, la investigación aporta a las PYMES que quieran utilizar políticas y estrategias para adquirir medidas de optimización de los indicadores financieros. Se establece el análisis de las cifras y se aporta con una explicación apropiada que sirve de guía. El aporte del presente estudio es útil a la academia ya que se puede estudiar las ventajas y desventajas de la aplicación de las normas internacionales financieras. Establece la generación del respectivo dialogo entre docentes y estudiantes con el fin de analizar las resultados principalmente económicos de la aplicación de las NIIF en PYMES de la ciudad de Ambato. 


\section{Sugerencias}

Las NIIF en las PYMES GRANDES de la ciudad de Ambato, se analiza el impacto socioeconómico de la adopción de las mismas a futuro. Es importante realizar un estudio a nivel de este tipo de empresas para establecer las implicaciones de la implementación en el ingreso neto, la comparación de PYMES con diversos sectores similares del cualquier lado del mundo le permitirá a la misma visualizarla como instrumento de análisis estadístico que servirá de base para investigar las características básicas entre similares entre las que se han internacionalizado o si puede proyectarse luego de la adopción de las NIIF.

\section{Implicaciones}

Para contribuir a la literatura esta investigación evaluó el estatus de la adopción de las NIIF pequeñas y los compara con el status de las NIIF completas Los informes que describen los requisitos y diferencias en la divulgación y otra información pertinente son realizados en base a las normativas contables. El objetivo de esta investigación ha sido estudiar y realizar una comparación del crecimiento de un sector de las PYMES de la ciudad de Ambato partiendo de la muestra de 560 empresas agrupadas en 25 áreas, asimismo permitieron evidenciar la simplicidad en la divulgación financiera para las PYMES. Se elaboraron las tablas que comparan las diferencias entre el año 2017 y 2018, así como también la elaboración de gráficos que se muestran en este estudio. Los datos que se incluyen en las tablas se obtuvieron de la base de datos de la Superintendencia de Compañías, Valores y Seguros.

\section{Conclusiones.}

\section{Gestión administrativa:}

- Las normas por ser esquemas internacionales de obligatoria aplicación, requieren de actualizaciones y capacitaciones constantes por parte del personal responsable. Las empresas tendrán que cumplir nuevos retos y tener la capacidad de adaptarse a un esquema y/o registros estándares que especifica la norma, con la finalidad de tener información contable transparente y oportuna.

- Estos retos no solo son parte del departamento financiero, sino que también involucra a la Gerencia con criterios estratégicos y organizacionales frente a la implementación de las NIIF en las Pymes de la ciudad de Ambato. Con la información obtenida de los periodos analizados permitió tener una mayor comprensión a la gestión que realizaron las PYMES. Con del esfuerzo, mancomunado entre administrativo, trabajadores, clientes y proveedores, se lograron tomar decisiones oportunas que fortalecen y consolida el crecimiento empresarial.

\section{Gestión financiera.}

- A partir de la adopción de la normativa NIIF, las empresas están en la obligación de presentar un juego completo de Estados Financieros como son el Balance General y 
Estado de Resultados Integral, Flujo de Efectivo, Estado de cambio en el patrimonio, y Notas a los Estados Financieros. Sin la obligatoriedad de adopción de las NIIF para PYMES las empresas solo presentaban dos Estados Financieros (Balance General y Estado de Resultados).. Siendo los estados de Resultados Integral y el flujo de efectivo los más complejos en la elaboración, ya que se incorporaron nuevas cuentas y partidas que afectaron notablemente las cifras de los Balances.

- Por lo que a pesar de que se debe aplicar normativas generales, cada PYME maneja sus políticas y circunstancias en el momento de transparenta la información financiera de su empresa. En el estudio financiero realizado a un sector de las PYMES de la ciudad de Ambato se pudo evidenciar algunos hallazgos financieros en la adopción de las NIIFS, especialmente en la precisión de sus estimaciones contables.. De la muestra estudiada, se observó un único resultado positivo que fue el indicador de rentabilidad del Patrimonio. Los demás indicadores financieros demuestran resultados decrecientes para este tipo de empresas, entendiéndose estos efectos por el cambio de normas que se revierte en las cuentas de resultados, y del balance general.

\section{Cuentas por cobrar:}

- La norma dice que se debería aplicar la medición de instrumentos financieros, usando una tasa de mercado para descontar en caso que no se haya establecido un valor al momento de la transacción. No se registran un porcentaje de intereses en este tipo de cuentas a pesar que en muchos casos son a largo plazo.

- En el estudio realizado a las PYMES, se determinó que los costos adicionales que estos tienen hasta llegar a su precio de venta no están siendo reconocidos como un activo, distorsionando así los valores razonables que deben tener, tal y como determina la normativa NIIF. En la contabilidad y bajo cualquier normativa el tema inventarios juega un papel muy importante.

- Activos fijos: La aplicación de las NIIFS en este grupo de cuentas es de gran relevancia, para el estudio, debido a la historicidad que estos mantienen. Mientras más antiguas sean, mayor será el ajuste que habrá que realizar. En el estudio se verifico que las empresas tuvieron que realizar avalúos, exenciones o depreciaciones, con la finalidad de transparentar las cifras y el registro sea a un valor razonable, como establece la normativa.

\section{Referencias Bibliográficas}

Acnur.org. (06 de Agosto de 2019). https://www.acnur.org/5c93e4c34.pdf. Obtenido de https://www.acnur.org/5c93e4c34.pdf: https://www.acnur.org/5c93e4c34.pdf 
Becerra, D. P. (2010). La globalización y el crecimiento empresarial a través de estrategias de internacionalización. Pensamiento y Gestión, No 28, 2.

Carrillo, s. (15 de Junio de 2019). https://blog.grupoenroke.com/que-son-laspymes\#: :text $=$ En\%20el\%20pa\%C3\%ADs\%20(Ecuador)\%2C, en\%20sus\%20proce sos\%20de\%20crecimiento. Obtenido de https://blog.grupoenroke.com/que-son-laspymes\#: :text=En\%20el\%20pa\%C3\%ADs\%20(Ecuador)\%2C,en\%20sus\%20proce sos\%20de\%20crecimiento.: $\quad$ https://blog.grupoenroke.com/que-son-laspymes\#: :text=En\%20el\%20pa\%C3\%ADs\%20(Ecuador)\%2C,en\%20sus\%20proce sos $\% 20$ de $\% 20$ crecimiento.

Chonchol, J. (1998). Impacto de la globalizacion en las sociedades latinoamericanas: ¿que hacer frente a ello? SCIELO, 1.

Diario el Telégrafo (28 de Junio de 2017). https://www.eltelegrafo.com.ec/noticias/economia/4/el-95-de-las-empresas-en-elpais-son-mipymes. Obtenido de https://www.eltelegrafo.com.ec/noticias/economia/4/el-95-de-las-empresas-en-elpais-son-mipymes: https://www.eltelegrafo.com.ec/noticias/economia/4/el-95-delas-empresas-en-el-pais-son-mipymes

Dir\&ge. (31 de Julio de 2018). https://directivosygerentes.es/pymes/noticias-pymes/pymesonu\#: : :text=Las\%20peque\%C3\%B1as\%20y\%20medianas\%20empresas, el\%2OPIB $\% 20 a \% 20$ nivel\%20global.\&text $=$ El\%20panorama\%20internacional\%20no\%20es,j uegan\%20en\%20la\%20econom\%C3\%ADa\%20mundial. Obtenido de https://directivosygerentes.es/pymes/noticias-pymes/pymes-

onu\#: :text=Las\%20peque\%C3\%B1 as\%20y\%20medianas\%20empresas,el\%20PIB $\% 20 \mathrm{a} \% 20$ nivel\%20global.\&text=El\%20panorama\%20internacional\%20no\%20es,ju egan $\% 20$ en $\% 201 \mathrm{a} \% 20$ econom\%C3\%ADa\%20mundial.:

https://directivosygerentes.es/pymes/noticias-pymes/pymesonu\#: :text=Las\%20peque\%C3\%B1 as\%20y\%20medianas $\% 20$ empresas,el\%20PIB $\% 20 \mathrm{a} \% 20$ nivel\%20global.\&text=El\%20panorama\%20internacional\%20no\%20es,ju egan\%20en\%201a\%20econom\%C3\%ADa\%20mundial.

Enrique Fowler Newton. (23 de Marzo de 2018). http://www.fowlernewton.com.ar/doctrinas/nic01.pdf. Obtenido de http://www.fowlernewton.com.ar/doctrinas/nic01.pdf: http://www.fowlernewton.com.ar/doctrinas/nic01.pdf

Garcia, D. M., \& Callejas, M. Y. (02 de Octubre de 2019). file:///C:/Users/emilio/Downloads/Dialnet-

CaracterizacionDeLasMPYMESEnLatinoamerica-2527677.pdf. Obtenido de file:///C:/Users/emilio/Downloads/Dialnet-

CaracterizacionDeLasMPYMESEnLatinoamerica-2527677.pdf:

file:///C:/Users/emilio/Downloads/DialnetCaracterizacionDeLasMPYMESEnLatinoamerica-2527677.pdf 
International Accounting Standards Board. (20 de Diciembre de 2012). https://www2.deloitte.com/co/es/pages/ifrs_niif/normas-internacionales-de-lainformacion-financiera-niif---ifrs.html\#: :text=Las\%20Normas\%20Internacionales\%20de\%20Informaci\%C3\%B3n, informaci\%C3\%B3n\%20a\%20revelar\%20sobre\%20las. Obtenido de https://www2.deloitte.com/co/es/pages/ifrs_niif/normas-internacionales-de-lainformacion-financiera-niif---ifrs.html\#: :text=Las\%20Normas\%20Internacionales\%20de\%20Informaci\%C3\%B3n,i nformaci\%C3\%B3n\%20a\%20revelar\%20sobre\%20las:

https://www2.deloitte.com/co/es/pages/ifrs_niif/normas-internacionales-de-lainformacion-financiera-niif---ifrs.html\#: :text=Las\%20Normas\%20Internacionales\%20de\%20Informaci\%C3\%B3n,i nformaci\%C3\%B3n\%20a\%20revelar\%20sobre\%20las

International Financial Reporting Standar. (19 de Marzo de 2018). https://www.ifrs.org/search/?query=seccion $+21+$ niif + para + pymes. Obtenido de https://www.ifrs.org/search/?query=seccion+21+niif+para+pymes: https://www.ifrs.org/search/?query=seccion+21+niif+para+pymes

Ley de Compañías. (29 de Diciembre de 2017). https://portal.compraspublicas.gob.ec/sercop/wpcontent/uploads/2018/02/ley_de_companias.pdf. Obtenido de https://portal.compraspublicas.gob.ec/sercop/wpcontent/uploads/2018/02/ley_de_companias.pdf: https://portal.compraspublicas.gob.ec/sercop/wpcontent/uploads/2018/02/ley_de_companias.pdf

Normas Internacionales de Información Financiera. (21 de Febrero de 2019). https://www.cyte.com.ec/niif-para-pymes/. Obtenido de https://www.cyte.com.ec/niif-para-pymes/: $\quad$ https://www.cyte.com.ec/niif-parapymes/

Stezano, F. (2013). Políticas para la inserción de lasmicroempresas y las pequeñas y medianas empresas en cadenasglobales de valor en AméricaLatina. México DF: Publicaciones de las naciones Unidas.

Stumpo, M. (11 D. de Octubre de 2018). https://repositorio.cepal.org/bitstream/handle/11362/44148/1/S1800707_es.pdf.

Obtenido de https://repositorio.cepal.org/bitstream/handle/11362/44148/1/S1800707_es.pdf: https://repositorio.cepal.org/bitstream/handle/11362/44148/1/S1800707_es.pdf

Superintendencia compañías, valores y seguros. (Noviembre de 13 de 2019). https://www.supercias.gob.ec/portalscvs/. Obtenido de https://www.supercias.gob.ec/portalscvs/: https://www.supercias.gob.ec/portalscvs/ 
Tápies, J. (20 de Junio de 2010). EMPRESA FAMILIAR NI TAN PEQUEÑA, NI TAN JOVEN. Barcelona: Inoc S.A. 
PARA CITAR EL ARTÍCULO INDEXADO.

López Samaniego, C. N., \& Naranjo Lozada, S. G. (2020). Impacto económico-financiero de la aplicación de las NIIFS en las PYMES de la ciudad de Ambato. Visionario Digital, 4(4), 98-116. https://doi.org/10.33262/visionariodigital.v4i4.1449

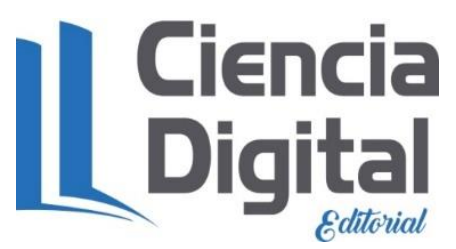

El artículo que se publica es de exclusiva responsabilidad de los autores y no necesariamente reflejan el pensamiento de la Revista Visionario Digital.

El artículo queda en propiedad de la revista y, por tanto, su publicación parcial y/o total en otro medio tiene que ser autorizado por el director de la Revista Visionario Digital.
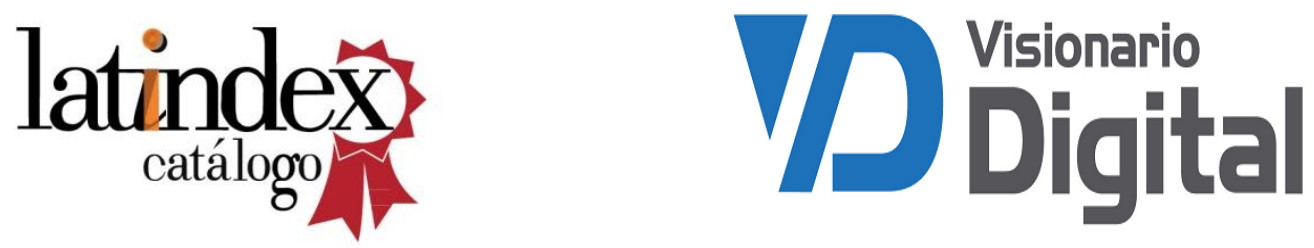